\title{
The Role of Hospitalists in Biocontainment Units: A Perspective
}

\author{
Maria G Frank, MD, FACP, SFHM ${ }^{1,2,3,4 *}$, Caroline Croyle, MPH, MPA, CPH 2,5 , Adam Beitscher MD $1,2,4$, Connie Price MD²,4
}

'Division of Hospital Medicine, Denver Health Hospital Authority, Denver, Colorado; ${ }^{2}$ Biocontainment Unit, Denver Health Hospital Authority, Denver, Colorado; ${ }^{3}$ Department of Medicine, Denver Health Hospital Authority, Denver, Colorado; ${ }^{4}$ Department of Medicine, University of Colorado School of Medicine, Aurora, Colorado; ${ }^{5}$ Department of Patient Safety \& Quality, Denver Health and Hospital Authority, Denver, Colorado.

n 2015, and in response to the Ebola virus outbreak in West Africa, the United States Department of Health and Human Services (HHS) designated 10 health departments and associated partner hospitals to become regional treatment centers for patients with highly infectious diseases, such as the Ebola virus and other highly infectious special pathogens (HISPs), and reinforce the nation's infectious disease response capability. These efforts catalyzed the creation and/or expansion of a network of biocontainment units (BCUs) to safely care for patients diagnosed with highly infectious diseases. These units are designed as special care units with environmental/engineering controls, laboratory capabilities, simple imaging testing, and dedicated staff to allow for the uninterrupted care of patients. ${ }^{1,2}$ The HHS approach closely resembled the tiered structure of trauma center levels familiar to the healthcare system. The regional framework identified four types of facilities (frontline healthcare facilities, assessment hospitals, treatment centers, and regional Ebola and other special pathogens treatment centers [RESPTCs]) with increasing levels of capabilities and responsibilities.

There are over 4,845 frontline healthcare facilities across the United States, which are able to identify and isolate a patient suspected of a HISP infection and inform local and state partners. The facility provides stabilizing treatment while coordinating the transport of the patient to a specialized center. An assessment hospital can identify and isolate a patient with a HISP, inform partnering agencies, and provide care at the facility for up to 96 hours. There are over 217 hospitals with this designation in the United States. Treatment centers are designated as state or jurisdiction treatment centers and have the capacity to care for HISP-infected patients for the entirety of their care plan, as well as serve as a partner in caring for a potential surge in high-risk patients if their partner RESPTC is unable to care for a patient because of capacity limits. Patients may receive care at a treatment center if and when it is determined to be more appropriate (eg, clinical purview, logistics, resources) than sending them to a RESPTC. There are currently 63 designated treatment centers in the United States.

As outlined by HHS, the RESPTCs ${ }^{3}$ must be ready to receive

*Corresponding Author: Maria G Frank, MD, FACP, SFHM; Email: maria.frank@ dhha.org; Telephone: 303-602-5011.

Published online first March 18, 2020

Received: November 14, 2019; Revised: February 14, 2020;

Accepted: February 23, 2020

() 2020 Society of Hospital Medicine DOI 10.12788/jhm.3402 a HISP-infected patient within their HHS region, domestically, or internationally within 8 hours. RESPTCs provide care for the entirety of the patient care plan. The 10 regional Departments of Public Health representatives are: Massachusetts (Region 1); New York (Region 2); Maryland (Region 3); Georgia (Region 4); Minnesota (Region 5); Texas (Region 6); Nebraska (Region 7); Colorado in partnership with Denver Health Hospital Authority (DHHA; Region 8); California (Region 9); and Washington State (Region 10).

\section{BCU PHYSICIAN STAFFING MODELS}

Most RESPCTs BCUs are staffed by a self-selected group of core providers with expertise in infectious diseases (ID) and critical care (CC). Teams are interdisciplinary and committed to a culture of safety. ${ }^{4-7}$ ID physicians are experts in HISP disease processes and epidemiology, which enables expert guidance on patient care and infection control. CC physicians are trained to provide care to patients requiring life-saving interventions.

\section{DHHA STAFFING MODEL}

As specialized units are shifting to include high-risk infectious diseases beyond Ebola, hospitals are developing innovative ways to manage a potential surge of patients with respiratory pathogens, expanding care far beyond the biocontainment unit. With the potential for an influx of HISPs in the healthcare setting, identifying stakeholders uniquely equipped to provide care in all areas of the hospital is ideal. At DHHA, the BCU physician staffing model transitioned from ID and CC physicians to a selected group of ten hospitalists as the primary managing service in 2018.

When DHHA received the RESPTC nomination, it developed a fully voluntary multidisciplinary high-risk infection team (HITeam; Table) with specialized training in personal protective equipment (PPE) donning and doffing, as well as BCU protocols. Our HITeam members participate in every-6-weeks mandatory team drills that involve practicing team dynamics and dexterity while wearing PPE. Team members participate in regular hospital and/or BCU-focused exercises to simulate and practice real-world experiences. They also participate in HISP Journal Club, where members from different disciplines discuss pertinent articles in the field, as well as every-other-month team-building meetings. Our unit's main challenges included maintaining competencies and staff retention. We developed a unique staffing model aimed at mitigating some of these challenges through a multidisciplinary team approach utilizing different levels of physician involvement. The first group 
TABLE. Members and Training/Skills Characteristics of Multidisciplinary HITeam

\begin{tabular}{lcc}
\hline Specialty/Provider Service & Number of Members in Hiteam & Special Training/Skills \\
\hline MD Hospitalists & 10 & $a-e, k, m$ \\
\hline MD Critical Care & 3 & $a-e, j, k, m$ \\
\hline MD Infectious Diseases & 4 & $a-e, k, m$ \\
\hline MD Leader (Medical Director) & 1 & $a-e, g, k-m$ \\
\hline Registered Nurse Leaders & 2 & $a, d, f-m$ \\
\hline Registered Nurses & 30 & $a, d, f-m$ \\
\hline Laboratory Technicians & 6 & $a, d, k, m$ \\
\hline Pharmacists & 2 & $d, k, m$ \\
\hline Respiratory Therapists & 2 & $a, c-e, g, j-m$ \\
\hline Paramedics & 14 & $a, c, m$ \\
\hline Infection Prevention Specialists & 2 & $a, d, g, k-m$ \\
\hline Emergency Management/Safety & 1 & $a, d, g, k-m$
\end{tabular}

apersonal protective equipment (PPE) skills

bpoint of care ultrasonography (POCUS)

cbedside procedures

abiocontainment unit (BCU) guidelines and protocols (available upon request)

${ }^{\circ}$ Conscious sedation and advanced airway management

fpediatric advanced life support (PALS)

gdecontamination

hbedside X-ray

'continuous renal replacement therapy (CRRT)

${ }^{\mathrm{j}}$ critical care training

kstandard institutional credentials

'waste management and decedent affairs

mhighly infectious special pathogens training applicable to discipline

comprises physicians providing primary, direct care to patients, which consists of our specially trained group of 10 hospitalists-including 2 pediatricians, as well as 3 CC and 4 ID consultants. The second group involves consultants from specialties such as nephrology, anesthesia, general surgery, radiology and gynecology; just-in-time training is available for them.

\section{WHY HOSPITALISTS?}

Hospitalists are uniquely positioned to care for this distinctive set of patients because they are comfortable with the care of acutely ill patients, many maintain bedside procedural skills, and many have acquired point-of-care-ultrasonography (POCUS) skills. Furthermore, hospitalists usually outnumber available specialists who may be needed to maintain consultative and critical care services for non-BCU patients. We were able to develop a feasible physician staffing model, as described below, and validate hospital medicine's commitment in addressing institutional needs.

In order to provide ideal unit coverage, 2 hospitalists are scheduled daily and available to respond in case of unit activation. $\mathrm{BCU}$ hospitalists are scheduled to cover the $\mathrm{BCU}$ when already scheduled to work a clinical shift. In the very infrequent event of BCU activation, BCU hospitalists would move their clinical work to the BCU and a back-up hospitalist would be called in to cover the "other" clinical shift; by overlapping coverage, we ensure BCU hospitalists' work-life balance and job satisfaction remain intact. Because of expected postexposure monitoring, individual physician's planned international travel is considered while generating the call schedule. When the unit is activated, the hospital provides payment for extra hours worked by both the BCU hospitalist and the back-up hospitalist, with anticipated revenue recapture through critical care billing by the BCU hospitalist. There are 10 HITeam hospitalists, who are trained and credentialed in bedside procedures and with different levels of POCUS expertise (from literacy to expert level). Team members are expected to attend training in bedside procedures while in PPE 2 times a year. The hospital also provides financial support for hospitalists seeking to further their BCU-related skills and training. It is anticipated that hospitalists will require consultative services from CC and ID in some selected cases to provide a well-rounded care approach to the biocontained patient.

\section{WHAT IT TAKES TO BE A TEAM}

Eligibility criteria for HITeam hospitalists include complete initial and quarterly maintenance PPE training; possession of 
active bedside procedure credentials for at least central line, paracentesis, thoracentesis, and arterial line; demonstration of basic POCUS skills, such as having enough POCUS verbiage literacy to be able to follow a radiologist's instructions on probe management (ie, a radiologist outside anteroom); and possibility of completing additional training and credentialing on conscious sedation and/or advanced airway management.

Similarly, hospitalists who join the team commit to attendance of at least one National Ebola Training and Education Center (NETEC) provider's course, representing the Region 8 RESPTC for educational presentations and research at regional and national levels, participation in quarterly HISP multidisciplinary meetings, attendance at quarterly donning and doffing sessions, as well as HITeam training sessions and drills, and active participation in the every-other-month HISP Journal Club/Grand Rounds to maintain competence and knowledge on management of many different pathogens, including Ebola and other filoviruses, MERS-CoV, SARS-CoV, Arenaviruses causing hemorrhagic fevers, Hantavirus, and novel influenzas or coronaviruses.

All of these commitments provide HITeam hospitalists with multiple opportunities for professional growth and development, such as augmenting scholarship venues by participating in collaborative national research projects, participating in national topic networks discussion groups and committees, becoming topic experts, and engaging in diversified training such as advanced airway training and conscious sedation. A new bedside ultrasound machine was purchased for the unit and housed within the division of hospital medicine with the intent to provide hospitalists the means necessary to achieve POCUS proficiency. Above all, by fostering a highly motivated and collegial multidisciplinary team, our model helps develop lasting partnerships at an institutional, regional, and national level.

This multidisciplinary team - with its skillfully trained and engaged nurses, physicians, respiratory therapists, pharmacists, infection control and laboratory specialists-works, learns, trains, and thrives collectively with the aim of providing excellent clinical care to our patients while assuring the safety of the team. DHHA has pioneered a RESPTC physician staffing model led by hospitalists.
We live in an ever-changing landscape of emerging diseases with blurred borders of disease geography. Hospitalists are versatile, capable of managing patients of varying acuity, able to perform many bedside procedures and POCUS; they are champions of interdisciplinary and teamwork disposition. By utilizing the resourcefulness of hospital medicine while helping to ease some of the burden that might otherwise be placed on a smaller numbers of physician groups, this approach provides a unique, cost-effective, and viable physician staffing model, which could be implemented in other BCUs in the United States.

Disclosures: Dr. Frank reports grants received outside the submitted work from (1) Hospital Preparedness Program - Ebola Preparedness (HPP-EP) grant, (2) Study Number MAPP-ZMAP-PRT-0077 an Expanded Access Treatment Protocol for ZMapp ${ }^{\text {TM }}$ for Patients with Zaire ebolavirus Disease (EVD) or Zaire ebolavirus (EBOV) exposure, from National Ebola Training and Education Center (NETEC) Supplement, and (3) Special Pathogen Research Network. All other authors have no conflict of Interest or funding to disclose.

\section{References}

1. Smith PW, Anderson AO, Christopher GW, et al. Designing a biocontainment unit to care for patients with serious communicable diseases: a consensus statement. Biosecur Bioterror. 2006;4(4):351-65. https://doi.org/10.1089/ bsp.2006.4.351.

2. Bannister B, Puro V, Fusco FM, Heptonstall J, Ippolito G; EUNID Working Group. Framework for the design and operation of high-level isolation units: consensus of the European Network of Infectious Diseases. Lancet Infect Dis. 2009;9(1):45-56. https://doi.org/10.1016/S1473-3099(08)70304-9.

3. US Department of Health and Human Services. Office of the Assistant Secretary for preparedness and Response Regional Treatment Network for Ebola and Other Special Pathogens. November 2017 Report. https://www. phe.gov/Preparedness/planning/hpp/reports/Documents/RETN-Ebola-Report-508.pdf Accessed August 17, 2019

4. Vasa A, Schwedhelm M, Johnson D. Critical care for the patient with Ebola virus disease: the Nebraska perspective. J Intensive Crit Care. 2015;1(8):1-5.

5. Garibaldi BT, Kelen GD, Brower RG, et al. The creation of a biocontainment unit at a tertiary care hospital. The Johns Hopkins Medicine experience. Ann Am Thorac Soc. 2016;13(5):600-608. https://doi.org/10.1513/AnnalsATS.201509-587PS

6. Beam EL, Boulter KC, Freihaut F, Schwedhelm S, Smith PW. The Nebraska experience in biocontainment patient care. Public Health Nurs. 2010 MarApr;27(2):140-7. https://doi.org/10.1111/j.1525-1446.2010.00837.x.

7. Hewlett AL, Varkey JB, Smith PW, Ribner BS. Ebola virus disease: preparedness and infection control lessons learned from two biocontainment units. Curr Opin Infect Dis. 2015 Aug;28(4):343-8. https://doi.org/10.1097/ QCO.0000000000000176. 